\title{
1. Zur Vorgeschichte der Gründung der Königlichen Biologischen Anstalt auf Helgoland
}

\author{
1.1. Erste Vorstellungen über die „Begründung“ einer kleinen \\ zoologischen Beobachtungs- und Untersuchungsstation an der \\ deutschen Nordseeküste und ihre nationale und internationale \\ Resonanz
}

Das Erscheinen von Charles Darwins ${ }^{1}$ Werk "The origin of species“ im Jahre 1859 veränderte das biologische Denken im 19. Jahrhundert und löste eine breite Bewegung des Evolutionismus in Wissenschaft und Weltanschauung aus. Noch 1790 hatte Immanuel Kant $^{2}$ in seiner "Kritik der Urteilskraft“ das Problem der Entstehung der Arten als unlösbar angesehen (vgl. Kant 1914). Er bezweifelte, daß ein neuer Isaac Newton ${ }^{3}$ auch nur die Erzeugung eines Grashalmes nach Naturgesetzen, die keine Absicht geordnet habe, erklären könne. Knapp hundert Jahre später wurde Darwin, der Kants Prognose widerlegt hatte, als hoch geehrter Wissenschaftler neben Newton in der Westminster Abbey feierlich beigesetzt.

In England wurde sein Buch von Wissenschaftlern wie Thomas Huxley ${ }^{4}$ bejubelt, aber auch bekämpft wie von Richard Owen ${ }^{5}$. Auch im Ausland wurde es mit großem Interesse aufgenommen. In Deutschland, welches damals in den biologischen Wissenschaften führend war, wurde das Werk mit besonderer Aufmerksamkeit bedacht, und Darwin erhielt große Zustimmung und Unterstützung von anerkannten Gelehrten, die mit Darwin korrespondierten. Einer der ersten „Darwinisten“ war Fritz Müller ${ }^{6}$, der 1864 sein Buch „Für Darwin“ veröffentlichte. Auch Emil du Bois-Reymond”, einer der Hauptvertreter der physikalischen Richtung in der Physiologie und Verfasser zahlreicher Werke auf verschiedenen Gebieten, gehörte zu den Anhängern Darwins. Seine Rede „Darwin und Kopernikus“ wurde als Gotteslästerung bekämpft. Wegen seines konsequenten Eintretens für den Darwinismus wurde du Bois-Reymond von Adolf Stöcker ${ }^{8}$, dem Gründer der Christlich-sozialen Partei, im preußischen Landtag heftig angegriffen, indem er seine Maßregelung forderte (vgl. Hörz u. Wollgast 1986). Auch August Weismann ${ }^{9}$, der in Freiburg wirkende Wegbereiter der modernen Genetik, war ein Verfechter von Darwins Entwicklungslehre. Er bekam ebenfalls die Ablehnung durch kirchliche Vertreter zu spüren. Als er seine Freiburger Antrittsvorlesung als "planmäßiger außerordentlicher Professor“ der philosophischen Fakultät zum Thema „Über die Berechtigung der Darwinschen Theorie“ hielt, blieben die Mitglieder der theologischen Fakultät demonstrativ fern (vgl. Uschmann 1965). Wie Weismann ein halbes Jahrhundert danach bekannte, hatte er jedoch auch später nie Grund gesehen, den Boden der Darwinschen Evolutionstheorie zu verlassen (vgl. Weismann 1909). Auch Anton Dohrn ${ }^{10}$ arbeitete auf dem Gebiet der Evolutionsforschung. Er

\footnotetext{
1 Charles Darwin (1809-1882)

2 Immanuel Kant (1774-1804)

3 Isaac Newton (1643-1727)

4 Thomas Huxley (1825-1895)

5 Richard Owen (1804-1892)

6 Fritz Müller (1822-1897)

7 Emil du Bois-Reymond (1818-1896)

8 Adolf Stöcker (1835-1909)

9 August Weismann (1834-1914)

10 Anton Dohrn (1840-1909)
} 
tauschte sich zwischen 1867 und 1882 mit Darwin über neue Erkenntnisse aus. Darwins Theorie beeinflußte die zoologische Forschung stark, weil sie die bereits vorliegenden umfangreichen empirischen Befunde in einen neuen Zusammenhang stellte. Die Evolutionstheorie brachte eine Vielzahl von Einzelfakten aus unterschiedlichen Gebieten wie Paläontologie, vergleichende Embryologie, vergleichende Anatomie, Systematik und Biogeographie in ein System. Diese Fächer, die vor Darwins Erkenntnissen Pfeiler des Schöpfungsglaubens waren, wurden durch den Darwinismus verändert. Was die vergleichende Embryologie anging, so beschäftigten sich die Zoologen vor allem damit, mit Hilfe der Untersuchung der Keimblätter die Beziehungen zwischen Wirbellosen und Wirbeltieren zu prüfen. Von besonderem Interesse waren jene Tierstämme, bei denen die Embryonalentwicklung völlig anders verläuft als bei den Wirbeltieren, die der Schwämme (Porifera), der radiärsymmetrischen Hohltiere (Coelenterata) und der zu den Articulata zusammengefaßten Ringelwürmern (Annelida) und Gliederfüßlern (Arthropoda) (vgl. Löther et al. 1982).

Der deutsche Zoologe Anton Dohrn gehörte zu den begeisterten Anhängern Charles Darwins. Er erforschte die Embryologie der Krebse, indem er rudimentäre Organe untersuchte. Sein Ziel war es, wie er in einem Brief an Darwin formulierte, zu einem „wirklichen

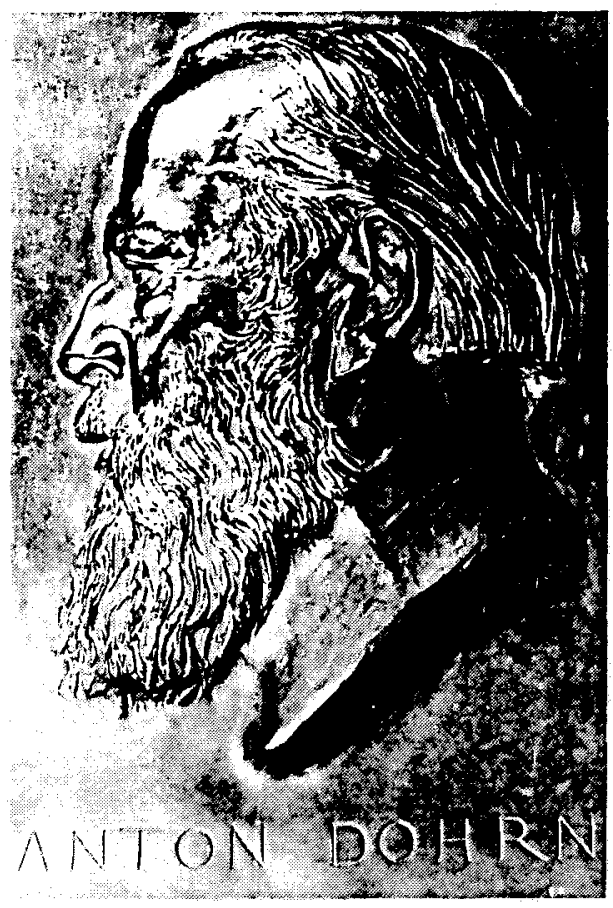

Abb. 1. Relief des Gründers der Zoologischen Station Neapel, Anton Dohrn, Reliefplastik, geschaffen von Adolf Hildebrandt um 1908. Anton-Dohrn-Archiv der Zoologischen Station Neapel. 
genealogischen System" zu kommen. Seine Untersuchungen hatte er sowohl am Mittelmeer als auch in der Nordsee, speziell in den Gewässern vor Helgoland, durchgeführt. Die Arbeitsbedingungen waren schwierig. Wie Anton Dohrn in einem Brief an Darwin im Dezember 1869 schreibt, habe er erfahren müssen, wie kompliziert es sei, embryologische Untersuchungen ohne Aquarium durchzuführen. Dies hätte ihn auf die Idee gebracht, nicht nur Aquarien, sondern ebenso Zoologische Stationen oder Laboratorien an verschiedenen Punkten der europäischen Küsten zu gründen (Dohrn 1869).

In diesem Sinne kann man davon ausgehen, daß die Gründung der ersten zoologischen Station mit dem Aufschwung verbunden war, den die biologischen Wissenschaften durch die Evolutionsforschung nahmen. Dohrn wurde von verschiedenen engagierten und einflußreichen Zoologen sehr stark unterstützt, so von Carl Vogt ${ }^{11}$. Vogt, der ab 1847 Professor in Gießen war, arbeitete sowohl anatomisch-physiologisch als auch paläontologisch. Der Wissenschaftler war ein eifriger Vorkämpfer für den Darwinismus, für den er in zahlreichen Schriften eintrat. Während der Revolution von 1848 wurde er als linksorientierter Politiker in die Frankfurter Nationalversammlung entsandt und 1850 wegen seines politischen Engagements seines Lehramtes in Gießen enthoben. 1852 erhielt er eine Professur für Geologie in Bern, später für Zoologie in Genf. Auch Carl von Siebold ${ }^{12}$ setzte sich leidenschaftlich für Dohrns Idee der Stationsgründung ein. Er wirkte ab 1885 als Professor in München und war Mitherausgeber der "Zeitschrift für wissenschaftliche Zoologie“. Er wurde vor allem durch seine taxonomischen und systematischen Arbeiten über Medusen („Quallen“) und Fische bekannt, so schrieb er ein „Lehrbuch der vergleichenden Anatomie der Wirbeltiere". Auch ein weiterer Befürworter, Ernst Haeckel ${ }^{13}$, ein Schüler von Johannes Müller ${ }^{14}$, gehörte zu den begeisterten Anhängern Darwins. Seine Forschungen galten besonders den niederen Meerestieren, so den einzelligen Strahlentierchen (Radiolaria), den Medusen der Nesseltiere (Cnidaria) und den Staatsquallen (Siphonophora), denen er große Monographien mit prachtvollen, schön gestalteten Tafeln widmete. Außerdem wurde er durch eine Vielzahl entwicklungsgeschichtlicher Werke bekannt, seine „Natürliche Schöpfungsgeschichte" von 1868 erreichte bis 192012 Auflagen und wurde in 15 Sprachen übersetzt.

Auch Darwin selbst war von Dohrns Idee begeistert und förderte die Einrichtung einer Station durch Geldspenden. Im Jahre 1872 wurde in Neapel eine der ersten zoologischen Stationen der Welt gegründet. Dohrn (1872) erläuterte in seinem Artikel „Der gegenwärtige Stand der Zoologie und die Gründung zoologischer Stationen" ausführlich, welche Bedeutung eine solche Einrichtung auf die sich entwickelnde Zoologie haben könnte, speziell für solche Gebiete wie Morphologie, Embryologie und Physiologie. Dohrns Anliegen war es, ein Forschungsinstitut zum „Studium der Lebensweise der Tiere" zu schaffen. Er wandte sich gegen „den Zerfall in das Kleinspezialistentum“ und das „museale Sammeln" (vgl. Dohrn 1872). Er bot an, Gelehrten die aufwendigen Expeditionsreisen zu ersparen und ihre Forschungsaufenthalte zu organisieren. Dazu gehörte auch die Bereitstellung von Versuchsmaterial und Laborräumen (vgl. Dohrn 1872). Viele Wissenschaftler arbeiteten vergleichend-beschreibend auf dem Gebiet der Entwicklungsgeschichte und nutzten dabei mikroskopische Labortechniken, die sie auch weiterentwickelten. Besonders diese Forscher waren auf große Mengen lebender Larven angewiesen und hatten Interesse an der Grün-

11 Carl Vogt (1817-1895)

12 Carl von Siebold (1804-1885)

13 Ernst Haeckel (1834-1919)

14 Johannes Müller (1801-1858) 
dung einer zoologischen Station. Hinzu kamen weitere organisatorische Probleme. So hatte die Mehrzahl der Wissenschaftler Schwierigkeiten, die Forschung mit ihren Lehrverpflichtungen an den Universitäten in Einklang zu bringen, besonders, da die notwendigen Expeditionen eine häufige Abwesenheit erforderten. Auch genügten die Arbeitsmöglichkeiten an den Universitäten nicht mehr den gestiegenen Anforderungen. Die Folge war, daß neben den hauptsächlich für die Lehre vorgesehenen Universitäten und Hochschulen gegen Ende des 19. Jahrhunderts neue Institutstypen entstanden, die meist auf privaten Stiftungen basierten. Dazu gehörten die biologischen Stationen, die in schneller Folge in ganz Europa entstanden. Tatsächlich machten u. a. in der Zoologischen Station Neapel die Begründer der experimentellen und entwicklungsphysiologischen Richtung unter dem Einfluß der Ideen Darwins ihre wichtigen Entdeckungen.

Die Zoologische Station Neapel war die berühmteste Einrichtung im Mittelmeergebiet, zu erwähnen sind jedoch außerdem vergleichbare Institutionen in Triest (1875), das französische Laboratoire Arago in Banyuls-sur-mer (1881) und Villefranche-sur-Mer (1881) an der französischen Mittelmeerküste. Auch an anderen Meeren wurden zoologische Stationen gegründet, so die schwedische Station Kristineberg (1877) und die britische Station Plymouth (1876). Die Umstände ihrer Gründung im einzelnen zu untersuchen, soll späterer Arbeit vorbehalten sein. Die Zoologische Station Neapel, die deutschen Stationsgründern immer wieder als Vorbild vorschwebte, war über ihre wissenschaftliche Bedeutung hinaus ein Zentrum kulturellen und künstlerischen Austauschs. Hier hielten sich nicht nur nahezu alle führenden Zoologen jener Zeit auf, sondern auch bekannte Künstler wie Paul $\mathrm{Klee}^{15}$, Arnold Böcklin ${ }^{16}$ und Fjodor Iwanowitsch Schaljapin ${ }^{17}$ (vgl. Groeben 1975). Unter den Zoologen wurde Wilhelm Waldeyer ${ }^{18} \mathrm{u}$. a. durch seine Arbeiten auf dem Gebiet der Zelltheorie bekannt, ihm verdanken wir die Einführung des Begriffs „Chromosom“. Franz Eilhard Schulze ${ }^{19}$, ab 1884 ordentlicher Professor für Zoologie und Direktor des neugegründeten Zoologischen Instituts in Berlin, beschäftigte sich vorrangig mit taxonomischen und entwicklungsgeschichtlichen Problemen, so dem Studium der marinen Schwämme. Carl Chun $^{20}$, ab 1898 ordentlicher Professor für Zoologie an der Universität Leipzig, war u. a. mit Arbeiten über Tintenfische (Cephalopoda) und Rippenquallen (Ctenophora) hervorgetreten und leitete die erste deutsche Tiefsee-Expedition. Victor Hensen ${ }^{21}$, ab 1868 ordentlicher Professor für Physiologie an der Universität Kiel, arbeitete u. a. über vergleichende Morphologie und Physiologie der Sinnesorgane. Im Jahre 1889 leitete er die große Atlantik-Plankton-Expedition der Humboldt-Stiftung, er prägte den Begriff „Plankton“. Einer der wenigen Kritiker Darwins war Wilhelm $\mathrm{His}^{22}$, ab 1872 ordentlicher Professor für Anatomie an der Universität Leipzig, später in Berlin. Er hat sich vor allem durch Einführung neuer Methoden in die Embryologie verdient gemacht, so erforschte er mittels verbesserter histologischer Techniken die Histogenese des Nervensystems. Weniger bekannt durch seine eigenen Veröffentlichungen, als vielmehr durch seine Kontroverse mit Anton Dohrn, wurde der Zoologe Robby August Kossmann ${ }^{23}$.

15 Paul Klee (1879-1940)

16 Arnold Böcklin (1827-1901)

17 Fjodor Schaljapin (1873-1938)

18 Wilhelm Waldeyer (1836-1921)

19 Franz E. Schulze (1840-1921)

20 Carl Chun (1852-1914)

21 Victor Hensen (1835-1924)

22 Wilhelm His (1831-1904)

23 Robby A. Kossmann (1849-1907) 
Wie Gutachten und Meinungsäußerungen zu entnehmen ist, empfanden es viele deutsche Wissenschaftler als großen Mangel, daß in jener Zeit noch keine einzige Süßwasseroder meeresbiologische Station in Deutschland existierte. Dies wurde um so mehr bedauert, als es auch in Deutschland eine Tradition auf dem Gebiet der Meereszoologie gab. So hatten deutsche Zoologen nicht nur an südlichen Küsten geforscht, sondern auch an der Nordseeküste. Hierbei hatte sich vor allem Helgoland als Standort bewährt.

Von den auf Helgoland vor Gründung der Königlichen Biologischen Anstalt bearbeiteten Forschungsthemen und -ergebnissen sollen folgende genannt werden: 1835 fanden Christian Ehrenberg ${ }^{24}$ und Rudolf Wagner ${ }^{25}$ als Verursacher des Meeresleuchtens den zu den Dinoflagellaten gehörenden Einzeller Noctiluca scintillans. Johannes Müller führte 1845 Untersuchungen über die Anatomie der Larven von Seesternen und Seeigeln durch, die zu einer genaueren Vorstellung über die Stachelhäuter (Echinodermata) führten. Rudolf Leuckart ${ }^{26}$ sowie der Assistent Rudolf Wagners Heinrich Frey ${ }^{27}$ widmeten sich 1846 den Coelenterata. Das Lanzettfischchen Branchiostoma lanceolatum wurde von Max Schultze ${ }^{28}$ entdeckt. Heinrich A. Pagenstecher ${ }^{29}$, Ilja I. Mecznikoff ${ }^{30}$ und Ernst Ehlers ${ }^{31}$ führten Arbeiten über Würmer durch, Carl Gegenbaur ${ }^{32}$ über Seescheiden (Ascidia). Richard Greeff ${ }^{33}$ forschte ebenfalls über Echinodermata. Rudolf Wagner, Ernst Taschenberg ${ }^{34}$ und Richard Böhm ${ }^{35}$ arbeiteten über Medusen und Polypen der Coelenterata. 1854 kam Ernst Haeckel erstmals als Schüler Johannes Müllers nach Helgoland. Er befaßte sich $u$. a. mit Einzellern (Protozoa) und Medusen. 1865 setzte Ernst Haeckel während eines gemeinsamen Aufenthalts mit Anton Dohrn seine Arbeiten über Medusenentwicklung fort.

Die Idee, in Deutschland eine solche Meeresstation zu gründen, wurde erstmals von einem Mann vorgetragen, der bis 1876 noch nicht in Neapel gearbeitet hatte, aber an der Nordsee regelmäßige Studien betrieb, dem Zoologen Ernst Ehlers. Sein Arbeitsgebiet waren meeresbewohnende Würmer, die Polychaeta. Er vertrat bis 1874 in Erlangen das Fach Zoologie und wurde dann Ordinarius in Göttingen. Gemeinsam mit einem Kollegen gab er eine Zeitlang die „Zeitschrift für wissenschaftliche Zoologie“ heraus. In einem Schreiben an Adelbert Falk ${ }^{36}$, von 1872-79 preußischer Kultusminister, legte Ehlers am 28. 6. 1876 den ersten Plan zur „Begründung einer kleinen zoologischen Station“ vor. Im Vordergrund seiner Argumentation stand das wissenschaftliche Interesse an einer solchen Einrichtung. Ähnlich wie in Neapel sollten Gelehrte Gelegenheit haben, an lebenden Meeresorganismen zu arbeiten. Ehlers ging jedoch in seiner Konzeption über die reine Grundlagenforschung hinaus und stellte eine Reihe praktischer Konsequenzen für die Fischereiwirtschaft in Aussicht. Darüber hinaus dachte er daran, die Verwaltung des Bades Norderney, wo er seinen Plan verwirklichen wollte, durch eine publikumswirksame

24 Christian Gottfried Ehrenberg (1795-1876)

25 Rudolf Wagner (1805-1864)

26 Rudolf Leuckart (1822-1898)

27 Heinrich Frey (1822-1890)

28 Max Schultze (1825-1874)

29 Heinrich A. Pagenstecher (1825-1889)

30 Ilja I. Mecznikoff (1845-1916)

31 Ernst Ehlers (1835-1925)

32 Carl Gegenbaur (1826-1903)

33 Richard Greeff (1829-1892)

34 Ernst Taschenberg (1818-1898)

35 Richard Böhm (1854-1884)

36 Adelbert Falk (1827-1900) 


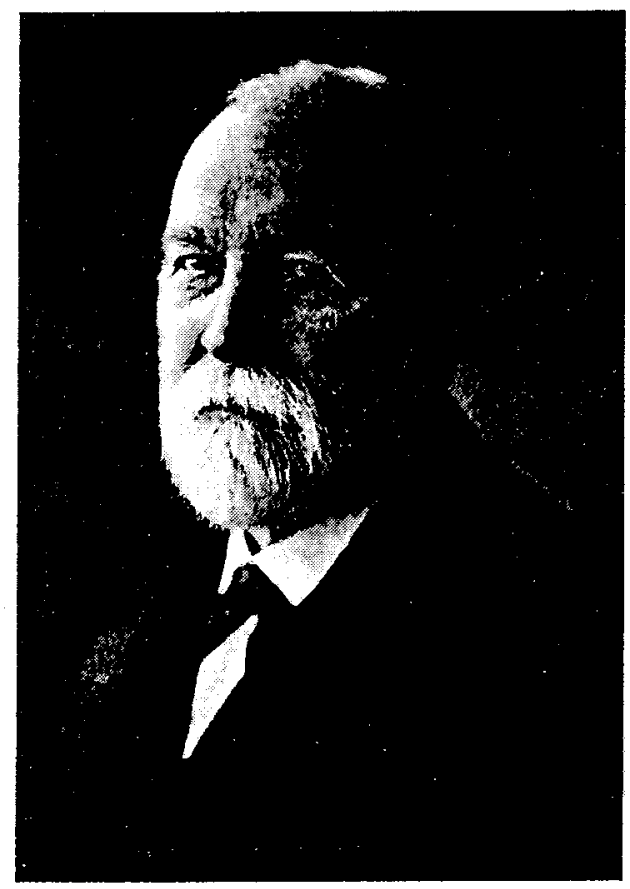

Abb. 2. Der Göttinger Zoologe Ernst Heinrich Ehlers (1835-1925), o. Prof. 1874, von 1881-1882 Prorektor der Georgia Augusta. Bildnisse von Göttinger Professoren aus zwei Jahrhunderten (1737-1937), Göttingen 1937.

Sammlung von Exponaten aus Fauna und Flora des Meeres für das Vorhaben zu interessieren. Was den rechtlichen Status anging, sollte sie, so Ehlers, „bei der provinciellen Zusammengehörigkeit und der Nachbarschaft von Norderney und Göttingen . . . als eine außerhalb des Universitätsverbandes stehende Filiale des Göttinger zoologisch-zootomischen Instituts angesehen werden" (86) und damit unter seiner Leitung stehen. Offensichtlich erhoffte sich der Wissenschaftler von diesem Vorstoß eine Erweiterung seiner Arbeitsmöglichkeiten.

Der Vorschlag, die zu gründende Einrichtung als „Filiale Göttingens“ anzusehen, erschien den Beamten im Kultusministerium so abwegig, daß sie diesen Passus in Ehlers' Schreiben mit einem roten Fragezeichen versahen.

Außer Norderney brachte Ehlers als Standort auch Helgoland ins Gespräch, verwarf jedoch den Gedanken wieder, da die Insel schwer zugänglich sei. Außerdem gehörte sie damals noch zu Großbritannien.

Nachdem sich Ehlers mit seinem Vorschlag an das Kultusministerium gewandt hatte, äußerte der Heidelberger Zoologe Robby August Kossmann auf der Hamburger Naturforscherversammlung im Herbst 1876 die gleiche Idee. Kossmann hatte u. a. bei Haeckel studiert und kannte Helgoland von Forschungsreisen her. Der Betrieb einer Meeresstation war ihm durch seine Tätigkeit an der Zoologischen Station Neapel von 1880-1881 vertraut. 
Ehlers, der seinen Vorschlag nicht öffentlich diskutiert hatte, betrachtete den Vorstoß Kossmanns jedoch nicht als Konkurrenz, sondern als Unterstützung seines Anliegens. Die Idee wurde von den anwesenden Wissenschaftlern begeistert aufgenommen. Die meisten Befürworter waren Zoologen, was mit dem Entwicklungsstand des Fachgebietes, seiner Breite und öffentlichen Anerkennung zusammenhing. Demgegenüber wurde die Botanik, vor allem die morphologisch-physiologische Richtung, weniger beachtet. Die Interessen der Botaniker wurden lediglich von Nathanael Pringsheim ${ }^{37}$ vertreten. $\mathrm{Er}$ war Mitbegründer der Deutschen Botanischen Gesellschaft und wirkte bis 1894 als ihr Präsident. Pringsheim setzte sich mit großem Nachdruck und Engagement für die Belange seines Fachgebietes ein. Aber auch Vertreter nichtbiologischer Disziplinen verwendeten sich von nun an für die Gründung einer biologischen Station in Deutschland, darunter Wilhelm von Freeden ${ }^{38}$, Ozeanograph und Gründer der Norddeutschen Seewarte, des heutigen Bundesamtes für Seeschiffahrt und Hydrographie. Von Freeden wurde u. a. als Verfasser eines Handbuchs der Nautik und als Herausgeber der Zeitschrift „Hansa. Zeitschrift für Seewesen“ bekannt.

Kossmann trug am 22. 9. 1876 sein Anliegen vor, noch am selben Tag wurde eine Kommission gegründet, die einen Entwurf zur „Errichtung zoologisch-botanischer Stationen an deutschen Meeren" erarbeiten sollte. Das Gremium präsentierte bereits am nächsten Tag eine Stellungnahme. Darin wurde betont, daß es gelte, die ehrenvolle Tradition der deutschen Zoologie, die sich in erster Linie auf Johannes Müller gründe, weiterzuführen.

Es wurden ausführliche Überlegungen zu der Frage angestellt, wo die Station einzurichten sei. Die Ausführungen wurden unter zwei wesentlichen Gesichtspunkten diskutiert, erstens des biologischen Formenreichtums, zweitens von der Durchführbarkeit des Planes. Viele Gründe sprachen für die Ostsee in der Nähe von Kiel. Kiel als Universitätsstadt bot bereits materiell und personell gute Voraussetzungen, die woanders erst hätten geschaffen werden müssen. Über den Standort aus wissenschaftlicher Sicht war man sich nicht sicher. Vor allem herrschte Unklarheit darüber, wie artenreich die Ostsee ist. Fest stand jedoch für die Wissenschaftler, daß die wenigen Gattungen und Arten reich vertreten seien und dieser Umstand zahlreiche Möglichkeiten für die Forschung eröffne.

Über Standorte an der Nordsee wurde ausführlich diskutiert, wobei man davon ausging, daß Forschung an beiden Meeren erwünscht und notwendig sei. Insofern sah man zwischen Kiel und einem Standort an der Nordsee keine Alternative, sondern die Ergänzung.

Was den Platz an der Nordsee anging, so wurde Helgoland vorgeschlagen. Man verwies darauf, daß die Insel nicht ohne Grund in den vergangenen Jahrzehnten Ort erfolgreicher zoologischer Forschung war. Sie biete mit ihrer außerordentlichen Vielfalt hervorragende Arbeitsmöglichkeiten. So könne man die Fauna felsiger Klippen studieren, die des Sandes, des Strandes und des offenen Meeres. Auch praktische Überlegungen flossen ein, die "stammverwandte" deutsche Bevölkerung sei mit der zoologischen Fischerei vertraut und habe schon in früherer Zeit wertvolle Hilfe geben können.

Zwar dominierten in der Diskussionsvorlage die Interessen der Zoologen, es wurde jedoch immer wieder betont, daß man den untrennbaren Zusammenhang der biologischen Wissenschaften im Auge habe und die einzurichtenden Stationen sowohl der zoologischen als auch der botanischen Beobachtung dienen sollten. Darüber hinaus wurden als wesentliche Aufgabe die physikalische und chemische Untersuchung des Meeres angesehen. Ziele der Einrichtung der Stationen waren:

37 Nathanael Pringsheim (1823-1894)

38 Wilhelm von Freeden (1822-1894) 
1. Erweiterung des Arbeitsfeldes für Zoologen und Botaniker;

2. Arbeitsmöglichkeiten für Gelehrte an deutschen Meeren zu schaffen (Einsparung von Zeit und Kosten);

3. Aufbau von Beziehungen zu den binnenländischen Lehranstalten;

4. Hebung des „Nationalwohlstandes“ durch Nutzung der an den deutschen Meeren gesammelten zoologischen Erkenntnisse für die Fischereiwirtschaft.

Einig war man sich darüber, daß sowohl beim Aufbau als auch beim Unterhalt der Station nur geringe Kosten entstehen dürften. Schon beim Bau der Gebäude sollte jeder „Luxus“ vermieden werden. Gedacht war an ein bescheidenes, einstöckiges Gebäude mit Aquarium im Souterrain sowie wenigen Wohn- und Arbeitsräumen. Die technische Ausstattung sollte auf das Nötigste beschränkt bleiben. Als Personal wollte man lediglich den Direktor und zwei Fischer beschäftigen. Außerdem hoffte man, unter den Gästen einen freiwilligen (vermutlich unbezahlten!) Assistenten zu gewinnen.

Es galt nun, staatliche Stellen für die Umsetzung dieser Ideen zu gewinnen. Die erste Schwierigkeit, die sich später als wesentlich erweisen sollte, tauchte bei der Frage auf, ob man das Projekt Preußen oder dem Reich unterstellen sollte. Angestrebt wurde, das Vorhaben zur „Reichssache“ zu machen. Taktisch wollte man folgendermaßen vorgehen:

Es sollte eine Denkschrift erarbeitet werden, die zur Information an das Reichskanzleramt, den Bundesrat, an die Regierungen der einzelnen Bundesstaaten sowie den Reichstag gesandt werden sollte. Man wollte die Königlich-Preußische Regierung bitten, die Federführung zu übernehmen. Die Denkschrift sollte geeignet sein, die Behörden vom Nutzen der Meeresstation zu überzeugen, und sie dazu bewegen, sich für das Projekt einzusetzen. Außerdem war geplant, eine größere Anzahl berühmter Gelehrter um ihre Unterschrift zu bitten, die dem Schreiben Nachdruck verleihen sollte. Für die Abfassung der Denkschrift sollten folgende namhafte Zoologen gewonnen werden: Ernst Haeckel (Jena), Rudolf Leuckart aus Leipzig, Pagenstecher (Heidelberg), und Julius Sachs ${ }^{39}$ aus Würzburg sowie der Botaniker Nathanael Pringsheim (Berlin). Auch der Kieler Kaufmann Heinrich A. Meyer ${ }^{40}$, der sich seit vielen Jahren mit Meeresbiologie befaßte, wurde einbezogen.

Der Versammlung wurden folgende vier Fragen zur Beschlußfassung vorgelegt:

1. Ob die Versammlung die Einrichtung zoologisch-botanischer Arbeitsstationen in Kiel und Helgoland als für die deutsche Wissenschaft notwendig erkläre.

2. Ob die Versammlung die Abfassung einer Denk- und Bittschrift für ein geeignetes Mittel halte, das Projekt durchzusetzen. Sie solle an das Reichskanzleramt, den Bundesrat und an die Regierungen der einzelnen Bundesstaaten sowie den deutschen Reichstag weitergegeben werden. Die Königliche Preußische Regierung solle gebeten werden, in dieser Sache die Initiative zu ergreifen.

3. Ob die Versammlung damit übereinstimme, die abzufassende Denkschrift einer noch zu erwählenden Kommission zu übertragen.

4. Ob die Versammlung unterstütze, die abzufassende Denkschrift einer größeren Anzahl von durch wissenschaftliche Leistung und Stellung berufenen deutschen Gelehrten vor der Überreichung zur Unterzeichnung zu unterbreiten (87).

Die Versammlung stimmte allen vier Punkten zu.

In der Denkschrift wurden die Vorstellungen der Wissenschaftler präzisiert. Demnach sollte die Station einerseits - wie die Zoologische Station Neapel - Gelehrten Arbeitsmöglichkeiten geben, andererseits sollte dort eigenständige Forschung betrieben werden. In der

39 Julius Sachs (1832-1897)

40 Heinrich A. Meyer (1822-1889) 


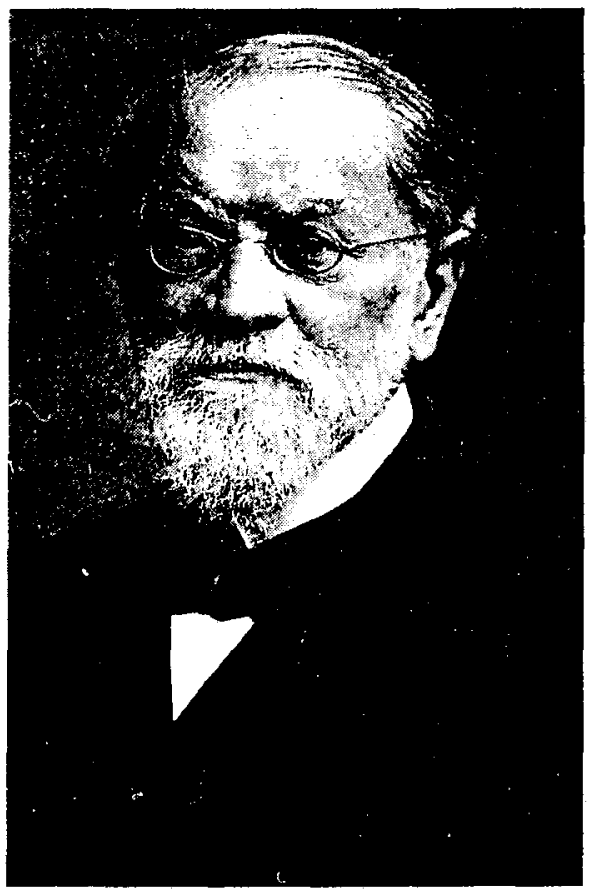

Abb. 3. Der Botaniker Nathanael Pringsheim (1823-1894), der sich Verdienste um die Gründung der Königlichen Biologischen Anstalt zu Helgoland erwarb. Archiv der Biologischen Anstalt Helgoland.

Präambel wurden zwei Anliegen hervorgehoben. Erstens wollte man sogenannte „reine Meeresbiologie" betreiben, d. h. die ganze Nordsee zoologisch-botanisch, chemisch und geologisch erforschen. Hierbei wollte man sich zunächst auf die Erkundung der näheren Umgebung von Helgoland konzentrieren. Zweitens sollte die zu gründende Anstalt durch ihre Arbeiten auf dem Gebiete der angewandten Meeresbiologie auch der deutschen Hochseefischerei nützen. Diese Aufgabe nahm in der Denkschrift den dominierenden Platz ein. Ausführlich wurde erläutert, daß man in diesem Zusammenhang die Erforschung der Fischgründe der Nordsee hinsichtlich ihrer Bodenbeschaffenheit, der gesamten Fauna und ihrer Produktion an Nutzfischen anstrebe. Geplant waren außerdem systematische Versuche zur künstlichen Aufzucht von Nutztieren (insbesondere Hummern). Schon damals dachte man daran, die Fischbestände zu schützen. So wollte man Untersuchungen anstellen lassen, die der Festsetzung von Schonmaßregeln und Schonzeiten von Nutzfischen dienen sollten. Was die Organisation der Arbeit angeht, so dachte man daran, neben Arbeitsplätzen für Angestellte der Station auch Gastarbeitsplätze für Forscher einzurichten. Um Fischer aus ganz Deutschland mit neuesten Erkenntnissen auf dem Gebiet der Fischwirtschaft vertraut zu machen, wollte man spezielle Kurse einrichten. Ähnlich wie die Zoologische Station Neapel sollte die deutsche Meeresstation wissenschaftliche Einrichtungen sowohl mit lebenden Seetieren als auch mit zoologischen und botanischen Präparaten versorgen. Dadurch erhoffte man sich hohe Einnahmen, die zum Unterhalt der Station beitragen sollten. Überhaupt widmeten sich die Verfasser der Denkschrift ausführlich Fragen der Finanzierung, da sie wußten, daß angesichts der angespannten wirtschaftlichen Lage 
davon hauptsächlich die Genehmigung des Projekts abhängen würde. Die einmaligen Ausgaben wurden vorsichtig auf 103000 Mark geschätzt, die laufenden Aufwendungen auf jährlich 33000 Mark (5).

Die Vorschläge setzten eine öffentliche Diskussion in Gang, in der unterschiedliche Meinungen zur Sprache kamen. Obwohl die meisten Zoologen das Projekt begeistert befürworteten, gab es auch Widersacher. Ein einflußreicher Gegner war Anton Dohrn. Er befürchtete eine starke Konkurrenz für die von ihm geleitete Neapler Station. Er hatte bereits schlechte Erfahrungen mit der 1881 gegründeten Station von Villefranche an der französischen Mittelmeerküste gemacht, die wegen der kürzeren und angenehmeren Reisewege und der besseren klimatischen Bedingungen immer mehr Zoologen anzog (vgl.I. Müller 1992). Damit wurde der Erhalt der Neapler Station, die hauptsächlich durch die Vermietung von Arbeitstischen finanziert wurde, gefährdet. Es kam hinzu, daß viele Mitglieder der Kommission zu den Sponsoren der Neapler Station gehörten und zu befürchten war, daß ihre Unterstützung für die Neapler Station zugunsten des deutschen Projekts nachlassen würde.

Um solche Bedenken zu zerstreuen, hatte die Kommission, die den Entwurf für die Errichtung einer deutschen Meeresstation erarbeitet hatte, ausdrücklich betont, daß man die hohe Bedeutung und Leistungsfähigkeit der Zoologischen Station in Neapel anerkenne. Man schätze die Großzügigkeit des Reiches und verschiedener Bundesstaaten, die die Arbeit in Neapel ermögliche, und sei weit davon entfernt, dieser schönen Anstalt ihre moralische Unterstützung und finanzielle Hilfen schmälern zu wollen.

Für Dohrn blieb das deutsche Vorhaben trotz der Versicherungen ein Konkurrenzunternehmen. In einem persönlichen Brief an seinen engsten Freund, den schottischen Dichter und Schriftsteller Charles Grant ${ }^{41,}$ schilderte Dohrn, wie er den Antrag aufgenommen hatte. Mit Verbitterung registrierte er, daß man die klimatischen und gesundheitlichen Verhältnisse Neapels während eines Teils des Jahres kritisiert hatte, aber noch mehr störte ihn der Antrag, die künftige Station auf Helgoland mit einer einmaligen staatlichen Unterstützung aufzubauen und durch regelmäßige staatliche Zuwendungen zu stützen.

Dohrns Einrichtung wurde teilweise aus einer Privatstiftung finanziert, aber ebenfalls staatlich gefördert. Dohrn sah deshalb seine eigenen Anträge auf Subventionierung gefährdet. Gegenüber Grant äußerte er den Plan, die Bemühungen um die Gründung einer Helgoländer Station zu torpedieren. Er kündigte an, „In wissenschaftlichen Kreisen eine Gegenströmung hervorzurufen $(\mathrm{zu})$ suchen und dadurch wenigstens die vorbereitenden Schritte des Comites zu verzögern“ (Dohrn 1876).

Nach Dohrns Aussagen beurteilten auch andere Wissenschaftler den auf der Naturforscher-Versammlung vorgetragenen Plan sehr kritisch. So schrieb der englische Embryologe Francis Balfour ${ }^{42}$ für „Nature“ einen abweisenden Artikel. Auch der Kieler Physiologe Victor Hensen soll sich nach Dohrns Aussagen negativ geäußert haben, indem er das Vorhaben "schädlich und thöricht“ nannte (154).

Die Diskussionen um die Stationsgründung wurden auch von persönlichen Auseinandersetzungen beeinträchtigt, in die Befürworter und Gegner der Stationsgründung verwickelt waren. Dohrn lag im Streit mit Professoren verschiedener Universitäten, die seine wissenschaftlichen Ansichten nicht teilten, darunter Carl Semper ${ }^{43}$ aus Würz-

41 Charles Grant (1841-1889)

42 Francis Balfour (1851-1882)

43 Carl Semper (1832-1893) 
burg, Carl Claus ${ }^{44}$ aus Göttingen sowie Carl Gegenbaur und Ernst Haeckel (132). Auch Kossmann war mit Dohrn verfeindet. Er machte ihm die Ansprüche auf Lösung einer zoologischen Fragestellung streitig (vgl. Kossmann 1875). Dohrn bekannte in einem Brief an seine Frau, daß er „ganz im Stillen“ dem Helgoland-Kiel-Projekt ein Bein nach dem anderen stelle, es sowohl in Potsdam beim Kronprinzen denunziert habe als auch im Unterrichtsministerium und bei Reichstagsabgeordneten. Auch einflußreiche Mitglieder der Königlich-Preußischen Akademie der Wissenschaften versuchte Dohrn gegen das Projekt aufzubringen, darunter den Physiologen du Bois-Reymond und den Geologen Justus Roth $^{45}$, Professor an der Friedrich-Wilhelms-Universität zu Berlin:

„Freund Kossmann soll merken, dass es noch Instanzen gibt, die sich dreimal besinnen, ehe sie seine Projecte unterschreiben ... Es ist vortrefflich, dass diese beiden (Justus Roth und Emil du Bois-Reymond) die Ursache der Kossmannschen Feindseligkeiten kennen, also von vornherein den Zusammenhang begreifen, in dem die Helgoland-Station damit steht" (155).

Aber nicht nur Befürworter und Gegner des Projekts standen sich gegenüber, auch innerhalb des Lagers der Befürworter offenbarten sich Gegensätze. Sie betrafen die Wahl des Ortes, vor allem für die Nordseestation, ihre staatliche Unterstellung und die wissenschaftliche Konzeption. Die Vorstellungen reichten von einer reinen Forschungsstation, die ausschließlich Grundlagenforschung betreiben sollte, bis zu einer Einrichtung, die auf die Fragen der Seefischerei ausgerichtet sein sollte. Auch eine Arbeitsstätte ohne eigenes Forschungsprofil für Forscher aus aller Welt war im Gespräch, vergleichbar der Zoologischen Station Neapel. Diese Auseinandersetzungen setzten sich noch bis 1891 fort und wurden besonders deutlich beim Streit um die personelle Besetzung der Station. Hinzu kamen, wie anläßlich der Gründung der deutschen Zoologischen Gesellschaft zum Ausdruck kommt, unterschiedliche Auffassungen darüber, ob die künftige Einrichtung dem Reich oder Preußen unterstellt werden sollte. Hintergrund waren die Spannungen zwischen Zoologen aus dem südwestdeutschen Raum und den spöttisch als „Berliner Oberzoologen“ bezeichneten Koryphäen wie Rudolf Leuckart, Karl Möbius ${ }^{4 \hat{6}}$ und Franz Eilhard Schulze. Möglicherweise spielte hier die umstrittene Vormachtstellung Preußens eine Rolle, mit der sich Wissenschaftler aus Süddeutschland nicht abfinden konnten.

Es gab auch abweichende Meinungen zur Erklärung der Naturforscherversammlung, darunter die von Ehlers, der im Juni 1876 als erster die Gründung einer deutschen Meeresstation vorgeschlagen hatte. Bereits im Oktober desselben Jahres konnte er umfangreiche Recherchen vorlegen, die er an der deutschen Nordseeküste angestellt hatte. Wie aus dem Bericht von Ehlers hervorgeht, hatte ihn das Ministerium dazu aufgefordert. Ehlers erläuterte, wie er sich die Verwirklichung seiner Idee vorstelle. Er äußerte sich eingehend über die Frage der Wahl des Standortes. Ihm erschienen Norderney und Borkum geeignet. Als Argumente führte er erstens die baulichen Möglichkeiten an und die Unterstützung durch ansässige Fischer. Er entwarf genaue Pläne, wie das Institut aussehen sollte, wie er sich die Arbeit dachte, welches Personal eingestellt werden könnte usw. Dem Vorschlag lag eine akribische Auflistung des Bedarfs an Geräten und Chemikalien bei (88). Die Sitzungsberichte der Naturforscherversammlung und einen befürwortenden Artikel Freedens hatte Ehlers zur Unterstützung seines Anliegens beigelegt. Ehlers ließ jedoch deutlich werden, daß durchaus Differenzen in der Wahl des Ortes bestanden, er bekräftigte den Wunsch, die Station auf Norderney zu errichten und seinem Institut anzugliedern,

44 Carl Claus (1835-1899)

45 Justus Roth (1818 -1892)

46 Karl Möbius (1825-1908) 


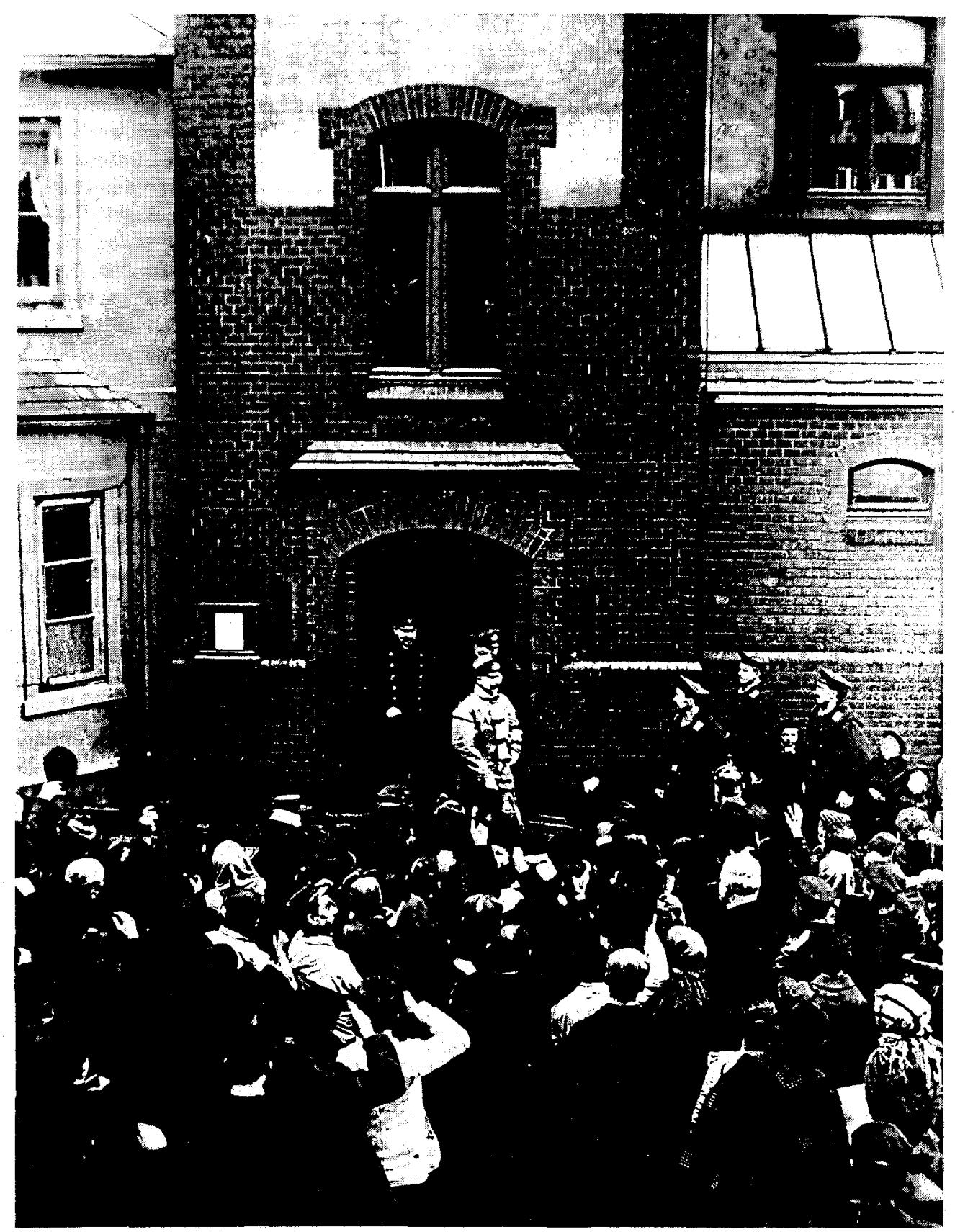

Abb. 4. Wilhelm II., Deutscher Kaiser und König von Preußen (1859-1941), auf Helgoland. Foto: Franz Schensky, Archiv der Biologischen Anstalt Helgoland. 
während die Wissenschaftler um Kossmann Helgoland favorisierten. Ehlers machte gegen Helgoland keine inhaltlichen Einwände geltend, sondern nur - wie erwähnt - die Tatsache, daß es zur britischen Krone gehöre und außerhalb der Badesaison schlecht erreichbar war. Ein weiterer Differenzpunkt war das von Ehlers ins Gespräch gebrachte Meeresmuseum. Er wollte die Gemeinde von Norderney für die Idee des Stationsbaus gewinnen, indem er ein von der Station betriebenes Museum als Attraktion für Gäste der Station in Aussicht stellte. Die Teilnehmer auf der Naturforscherversammlung zogen diesen Vorschlag auch in Erwägung, dachten jedoch daran, Aquarium, Versand und Museum der Privatindustrie zu überlassen.

Einigkeit im Anliegen, aber Streit in allen anderen Fragen, die Standort und Arbeit der Station an der deutschen Nordseeküste betrafen, das war der Stand der Auseinandersetzungen um die erste deutsche Meeresstation in den Jahren 1876-90. Die Diskussion erhielt neuen Aufwind durch das Bestreben, die großen Probleme der deutschen Fischereiwirtschaft zu lösen. Mit staatlicher Unterstützung war zu rechnen, hatte sich doch unter dem Protektorat des Kronprinzen Friedrich Wilhelm der Deutsche Fischerei-Verein gegründet, aus dem 1885 als Zweig der Deutsche Seefischerei-Verein hervorging. Er wurde anfangs „Section für Küsten- und Hochseefischerei" genannt (vgl. Ehrenbaum und Lübbert 1913). Walther Herwig ${ }^{47}$, Gründer des Vereins und langjähriger Präsident, gilt als „Vater“ der Seefischerei. Als bahnbrechend sind seine Arbeiten zur Entwicklung der deutschen Heringsfischerei zu bezeichnen. Der Verein arbeitete eng mit Wissenschaftlern zusammen und organisierte z. B. Fischereiexpeditionen, an denen Wissenschaftler wie Karl Möbius und Victor Hensen teilnahmen (vgl. Meyer-Warden 1970). Herwig unterstützte junge Leute mit neuen Ideen und kümmerte sich auch um Friedrich Heincke ${ }^{48}$. Mit ihm gewann Herwig einen engagierten Mitstreiter für die Belange der deutschen Seefischerei, der nicht nur wissenschaftliche Beiträge leistete, sondern sich auch wissenschaftsorganisatorischen Fragen widmete (vgl. Ehrenbaum 1922).

Ein erster Schritt zur Stationsgründung war die im Jahre 1888 errichtete zoologische Wanderstation in der Ems-, Weser- und Elbemündung. Dort wirkte Ernst Ehrenbaum ${ }^{49}$, der später an der Königlichen Biologischen Anstalt zu Helgoland als Kustos arbeitete (vgl. Mollenhauer und Lüning 1988).

Über die Errichtung einer festen biologischen Station auf Helgoland wurde erst wieder diskutiert, als Helgoland dem Deutschen Reich angegliedert wurde.

\subsection{Der Sansibar-Vertrag - eine Voraussetzung für die Gründung der Königlichen Biologischen Anstalt auf Helgoland}

Im Mittelpunkt der öffentlichen Argumentation stand die strategisch günstige Lage der Insel. An der Rückführung des ehemals zu Schleswig-Holstein gehörenden Eilands bestand besonderes Interesse. Otto von Bismarck ${ }^{50}$ hatte schon lange zuvor an den Erwerb Helgolands gedacht, weil die Insel als unentbehrlich für die Verteidigung der deutschen Nordseeküste eingeschätzt wurde. Die Bedeutung Helgolands für Deutschland, so wurde in Zeitungsartikeln betont, liege besonders in seiner Lage als vorgeschobener Posten im Meere, außerdem sei der südliche Teil der Insel für eine schnelle Mobilmachung der Flotte geeignet.

47 Walther Herwig (1838-1912)

48 Friedrich Heincke (1852-1929)

49 Ernst Ehrenbaum (1861-1942)

50 Otto von Bismarck (1815-1898) 
In seiner Rede an die deutsche Marine machte der Deutsche Kaiser und König von Preußen Wilhelm II. am 10. 8. 1890, als er die Insel offiziell übernahm, folgendes deutlich:

„Das Eiland ist dazu berufen, wie ein Bollwerk zur See zu werden, den deutschen Fischern ein Schutz, ein Stützpunkt für meine Kriegsflotte, ein Hort und Schutz für das deutsche Meer gegen jeden Feind, dem es einfallen sollte, auf demselben sich zu zeigen" (Wilhelm II. 1890).

Mit einer ähnlich lautenden Begründung bestätigte das Abgeordnetenhaus am 26. 1. 1891 einstimmig den Gesetzentwurf über die Vereinigung der Insel Helgoland mit der preußischen Monarchie. Im Protokoll hieß es:

„Unerwünscht war es dem Deutschen, dem Preußen, daß vor den Mündungen unserer Ströme ein fremder Besitz in einer deutsch sprechenden zu Deutschland gehörigen Insel bestand. Nunmehr hat unser König diese Insel für Preußen gewonnen und wenn aus der Ferne Reisende, Deutsche, Preußen über ferne Meere dem Vaterlande wieder zusteuern, dann werden sie mit Freude die deutschen und die preußischen Fahnen von der Insel wehen sehen als ein Symbol der Macht und Ehre des wiederhergestellten Deutschen Reiches und Preußens" (2).

Am 1. 4. 1890 wurde die Insel von der englischen Krone übergeben, am 10. August nahm Wilhelm II. die Insel offiziell in Besitz. Der 10. August 1890 wurde von nun an als Gedenktag in der Geschichte gefeiert. Damit sei, so hieß es, eine Epoche des beständigen Wachstums, der Vergrößerung der Bevölkerung im Sinne des Kurfürsten, abgeschlossen. Zur Erinnerung an den Besuch des deutschen Kaisers und preußischen Königs auf der Insel wurde ein Denkmal errichtet und eine Steinplatte an der Stelle in den Boden eingelassen, wo der Kaiser an Land gegangen war.

Helgoland bildete von nun an eine Landgemeinde. Es wurde zwar unter die staatliche Verwaltung der Provinz Schleswig-Holstein und Süd-Dithmarschen gestellt, blieb aber kommunal selbständig. Obwohl Reichsgesetze in Helgoland eingeführt wurden, akzeptierte der Staat weiterhin bisher auf der Insel geltende Gepflogenheiten (136). So wurde festgelegt, daß die Verfassung der Gemeinde durch ein Statut bestimmt werden sollte, das vom Minister des Innern nach Anhörung von Alteingesessenen und des Kreisausschusses erlassen wurde. Wie es der Kaiser zugesichert hatte, stand Helgoland außerhalb der deutschen Zolleinheit, eine Regelung, die in späteren Jahren immer wieder umstritten war, aber dennoch beibehalten wurde. Außerdem sicherte der Regent die Befreiung von der Wehrpflicht für die unter britischer Herrschaft geborenen Personen zu und den Weiterbestand aller Vermögensrechte, die Personen oder Kooperationen der britischen Regierung gegenüber an Helgoland erworben hatten. Die Akzeptanz englischer Verträge hatte weitreichende Konsequenzen für den Ankauf bzw. die Pacht von Land und Gebäuden durch den Fiskus.

Der juristische Akt der Übernahme wurde durch Stellungnahmen von Wissenschaftlern begleitet. Sie beeilten sich, den Anspruch auf Helgoland „wissenschaftlich“ zu begründen.

Man berief sich auf zwei Argumente, die Geologen und Philologen einbrachten. Erstens sei Helgoland ein „versprengtes Stück deutschen Bodens“. Durch die Rückführung an Deutschland werde deshalb ein Zustand wiederhergestellt, der seit dem Ende der paläozoischen Zeit fast ununterbrochen bestanden habe. Zweitens sei die Helgoländer Bevölkerung deutschstämmig. Englische Historiker waren anderer Meinung darüber und behaupteten, es handele sich nicht um „richtige Deutsche“, sondern um „friesische Dänen“. So wurde - auch in der Presse - eine heftige Auseinandersetzung geführt. Die Tatsache, daß Fauna und Flora der Insel und ihrer Umgebung von deutschen Wissenschaftlern erforscht worden waren, wurde nicht erwähnt. Auch die Absicht, auf Helgoland eine Meeresstation 
zu gründen, spielte bei der Übernahme durch Wilhelm II. nur eine untergeordnete Rolle. Sie wurde in einigen Artikeln überhaupt nicht, in anderen nur in einem Halbsatz genannt.

Die Zoologen und Botaniker, denen die Stationsgründung am Herzen lag, versuchten, das politische Interesse an Helgoland für ihre Zwecke zu nutzen. Das Versprechen Wilhelms II, die Helgoländer Bevölkerung als jüngstes und liebstes Kind Deutschlands besonders zu fördern, wurde dahingehend interpretiert, daß die Errichtung einer Meeresstation eine solche Förderungsmaßnahme sei, auf die die Helgoländer ein Recht hätten. Dahinter stand die Erfahrung, daß - besonders im ausgehenden 19. Jahrhundert - wissenschaftliche Vorhaben besonders dann gefördert wurden, wenn sie politisch oder wirtschaftlich begründet werden konnten. Im Falle der Helgoländer Station wurde es üblich, politisches und wissenschaftliches Interesse miteinander zu verknüpfen. Heincke, der erste Direktor der 1892 gegründeten „Königlichen Biologischen Anstalt auf Helgoland“, drückte dies so aus:

„Das ist der hohe Wert Helgolands für die Wissenschaft, für die Erforschung großer Probleme des Lebens im Meer und in der Luft. Es verdient wohl, jener Bedeutung an die Seite gestellt zu werden, die unsere Insel als Marinefort und Signalturm für den Schutz der vaterländischen Küsten besitzt“ (vgl. Heincke 1899).

\subsection{Die Gründung der Königlichen Biologischen Anstalt auf Helgoland}

Bereits vor der offiziellen Angliederung Helgolands an Deutschland, die 1887 vereinbart worden war, nahmen die Wissenschaftler ihre Bemühungen um die Gründung der ersten deutschen Meeresstation wieder auf. Sie versuchten, Wilhelm II. für ihr Vorhaben zu interessieren, indem sie ihn darauf hinwiesen, daß der Neuerwerb Helgolands auch von großer wissenschaftlicher Bedeutung sei. Diese Mitteilung wurde vom Zivilkabinett mit Interesse zur Kenntnis genommen, wie aus einem Aktenvermerk hervorgeht, aber nicht beantwortet. Das Schreiben wurde „abgelegt“.

Wie so oft, ist die Verwirklichung des Projekts auf die Initiative eines einzelnen Mannes zurückzuführen, eines Mitarbeiters im Preußischen Kultusministerium. So ist es als Glücksumstand zu bewerten, daß sich Friedrich Althoff ${ }^{51}$ für die Gründung einer biologischen Station auf Helgoland engagierte. Der Straßburger Professor war ab Oktober 1882 als Vortragender Rat und Dezernent für die Personalangelegenheiten der Universitäten ins Kultusministerium aufgerückt. Sechs Jahre später wurde er Oberregierungsrat. Am 14. 4. 1897 wurde ihm als Ministerialdirektor die Leitung der Ersten Unterrichtsabteilung des Kultusministeriums übertragen. Hier war er einer von vier Ministerialdirektoren, zuständig für Hochschulen, wissenschaftliche Anstalten, Bibliotheken, Kunst, Museen, Denkmalspflege und mit der Ernennung zum Vorsitzenden der wissenschaftlichen Deputation für das Medizinalwesen am 1. Oktober 1900, auch für den außeruniversitären Bereich der Medizin. Am 10. 10. 1907 schied er aus dem Ministerium aus. Friedrich Althoff, von Zeitgenossen als „Preußens heimlicher Kultusminister“ bezeichnet, gilt zu Recht als einer der hervorragenden Förderer der Wissenschaften und Künste um die Jahrhundertwende. Zu seinen zahlreichen Interessen gehörte auch die Meeresforschung (vgl. Sachse 1928). Ihm ist es zu verdanken, daß im Jahre 1892 die Königliche Biologische Anstalt auf Helgoland als selbständige wissenschaftliche Institution gegründet werden konnte. Hierbei konnte sich Althoff auf engagierte Wissenschaftler stützen, die sich für die Stationsgründung ein-

51 Friedrich Althoff (1839-1908) 
setzten. Beim Studium der Akten wird deutlich, daß dieser langiährige, mühevolle Prozeß sowohl von seiten der Wissenschaftler als auch von der des Staates viel Geduld erforderte. Vor allem die Wissenschaftler wurden nicht müde, über Jahrzehnte hinweg ihre Argumente zu wiederholen. In nachstehender Tabelle sind die einzelnen Schritte dieser Abstimmung zwischen Staat und Wissenschaftlern chronologisch aufgelistet worden und sollen im Anschluß näher erläutert werden:

Tabelle 1. Übersicht über wichtige Schritte zur Gründung der Königlichen Biologischen Anstalt auf Helgoland zwischen 1890-92

\begin{tabular}{|ll|}
\hline Datum & Ereignis \\
\hline 16. 07. 1890 & Berufung einer Kommission zur Stationsgründung \\
18. 07. 1890 & Einzelgutachten von F. E. Schulze \\
21. 07. 1890 & Schreiben Pringsheims an Althoff \\
22.07.1890 & Antrag Pringsheims und F. E. Schulzes an die \\
& Königlich-Preußische Akademie der Wissenschaften \\
20.11.1890 & Gutachten der Königlich-Preußischen Akademie der Wissenschaften \\
Dez. 1890 & Stellungnahme der Deutschen Zoologischen Gesellschaft \\
27. 05. 1891 & Sitzungen im Kultusministerium zur Gründung der \\
& Königlichen Biologischen Anstalt auf Helgoland \\
15. 03. 1892 & Bestätigung des Etats durch das Preußische Herrenhaus \\
31. 05. 1892 & Erwerb des ersten Stationsgebäudes \\
\hline
\end{tabular}

Die Initiative, die Bemühungen um die Stationsgründung wiederaufzunehmen, gingvon Nathanael Pringsheim aus. Pringsheim hatte auf Helgoland wichtige Erkenntnisse gewonnen. Erstmals hatte er sich 1852 auf der Insel aufgehalten, wo er seine morphologisch-physiologischen Arbeiten an Meeresalgen durchgeführt hatte. Diese Forschungen setzte er während seiner Aufenthalte zwischen 1855 und 1862 fort. 1873 veröffentlichte er seine Ergebnisse. Pringsheim war schon krank und betagt und hatte besonderes Interesse daran, daß seine Arbeiten von jüngeren Kollegen fortgesetzt wurden. Er engagierte sich sehr für das Zustandekommen der Station und investierte sehr viel Kraft in die wissenschaftsorganisatorische Vorbereitung. Er hatte während der häufigen Aufenthalte persönliche Bindungen an die Insel gewonnen, die Friedrich Heincke wenige Jahre später so charakterisierte:

„Keiner der zahlreichen Naturforscher, die Helgoland besucht haben, hat so lebendiges und dauerhaftes Interesse, keiner so tiefe Zuneigung für unsere Insel gezeigt wie Pringsheim, keiner hielt soviel von ihrer biologischen Bedeutung wie er, keiner ist mit solcher Kraft und Wärme für die Errichtung einer biologischen Station hier eingetreten" (Heincke 1899).

Pringsheims Engagement hatte deshalb soviel Gewicht, weil er eine sehr angesehene Persönlichkeit war. Bereits im Jahre 1860 war er als Ordentliches Mitglied in die Königlich-Preußische Akademie der Wissenschaften berufen worden. Grundlage für diese Ehre waren jene Arbeiten, die er auf Helgoland angefertigt hatte. Der Begründer der Mikropaläontologie Christian Gottfried Ehrenberg, Professor der Medizin an der Berliner Friedrich-Wilhelms-Universität, würdigte die Leistungen Pringsheims. Mit der Wahl Prings- 


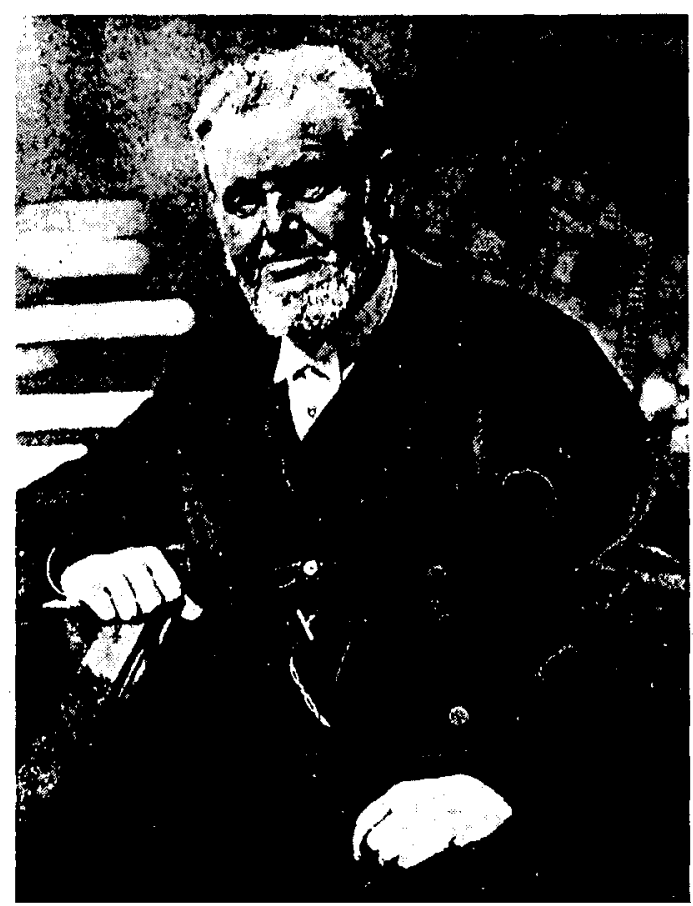

Abb. 5. Friedrich Althoff (1839-1908), ,heimlicher Kultusminister “ von Preußen. Archiv der Akademie der Wissenschaften Berlin, Fotosammlung.

heims wollte man, wie es Ehrenberg ausdrückte, die bisher vernachlässigte empirische Pflanzenphysiologie fördern.

Auf Initiative von Pringsheim wurde am 16. Juli 1890 eine Kommission gegründet, die sich der Stationsgründung widmen sollte. Zu diesem Zeitpunkt gehörte die Insel bereits zu Deutschland, war aber noch nicht offiziell durch Wilhelm II. übernommen worden. Außer Pringsheim gehörten der Kommission die Akademiemitglieder Franz Eilhard Schulze, Wilhelm Waldeyer und Karl Möbius sowie der Sekretär der physikalisch-mathematischen Klasse der Königlich-Preußischen Akademie der Wissenschaften, Emil du Bois-Reymond, an. Die Wissenschaftliche Kommission für Meeresforschung war durch ihren Vorsitzenden Herwig vertreten. Von seiten des Kultusministeriums nahm Friedrich Althoff teil.

Obwohl in früheren Jahren umfangreiche Stellungnahmen und Begründungen für die Stationsgründung erarbeitet worden waren, wurden die Wissenschaftler erneut aufgefordert, alles zu wiederholen.

Außerdem ging es darum, konzeptionelle Klarheit zu erreichen. Die größte Schwierigkeit bestand für Althoff darin, das Finanzministerium zur Bewilligung der Mittel zu bewegen.

Neben der Erarbeitung von Konzeptionen und der Einholung von befürwortenden Einzelgutachten war es wichtig, angesehene Gremien zur Unterstützung zu gewinnen. Ein solches im preußischen Staat sehr geschätztes Gremium war die Königlich-Preußische Akademie der Wissenschaften. Nathanael Pringsheim und Franz Eilhard Schulze, die beide der Akademie als ordentliche Mitglieder angehörten, ergriffen auch hier die Initia- 


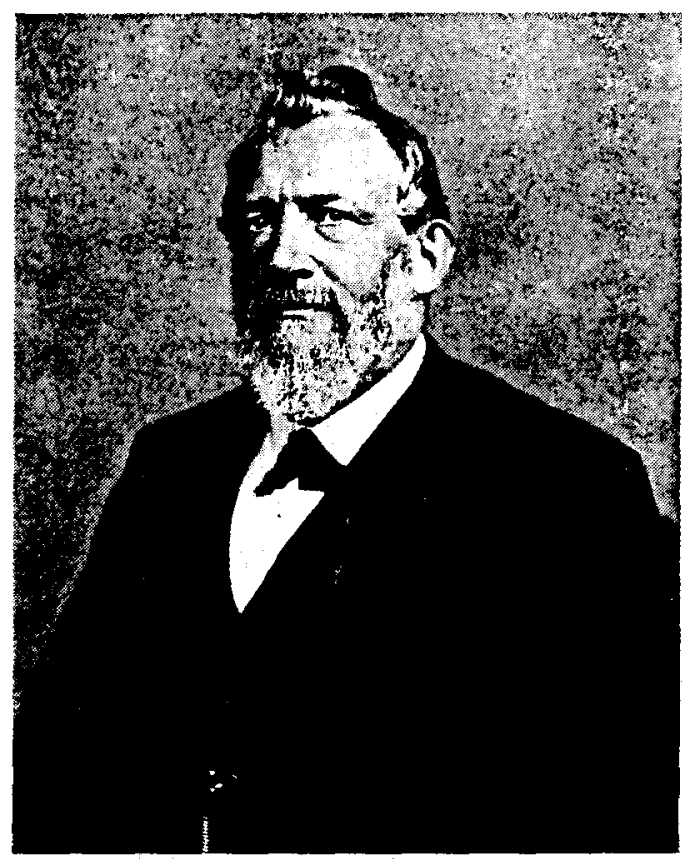

Abb. 6. Emil du Bois-Reymond (1818-1896), machte sich ebenfalls verdient um die Gründung der Königlichen Biologischen Anstalt auf Helgoland. Archiv der Akademie der Wissenschaften Berlin,

tive. Außerdem gewannen sie Richard Hertwig ${ }^{52}$. Sie stellten mit Schreiben vom 22. 7. 1890 bei der Königlich-Preußischen Akademie der Wissenschaften den Antrag, ein Gutachten über die Einrichtung einer biologischen Station auf Helgoland zu erstellen.

Dieses Gutachten wurde erarbeitet und im Plenum der Akademie, einer gemeinsamen Sitzung der physikalisch-mathematischen und der philosophischen Klasse, diskutiert. Es wurde eine zustimmende Stellungnahme verabschiedet. Darin heißt es:

„Mit Genugtuung begrüßt die Akademie die in der vorliegenden Eingabe geplante Gründung einer biologischen Station an unseren deutschen Meeren" (143).

Außer der Stellungnahme wurde noch ein ausführliches Gutachten angefertigt, das von allen Mitgliedern der beiden Klassen unterzeichnet wurde. Das Gutachten selbst wurde im November 1890 fertiggestellt (144). In dem Schriftstück wurde daran erinnert, daß die Akademie ein solches Vorhaben schon jahrelang unterstützt habe und mit den Motiven einverstanden sei. Deutlich wird das Bestreben, Forschungsprogramm und Personalpolitik zu beeinflussen. Die Gelehrten der physikalisch-mathematischen Klasse baten sich bei der Organisation und wissenschaftlichen Leitung der Einrichtung beratenden Einfluß aus. Auch bei der personellen Besetzung wollte man mitreden und äußerte sich sogar zu Gehaltsfragen. Vor dem Hintergrund der wirtschaftlichen Schwierigkeiten Deutschlands bestand die Sorge, daß die Station in den Dienst kurzfristiger ökonomischer Interessen gestellt werden könnte. Um dies zu vermeiden, setzten sich die Unterzeichner für ein

52 Richard Hertwig (1850-1937) 
breites Forschungsgebiet ein und schlugen vor, neben den Arbeiten, die im Interesse der Seefischerei lagen, auch Forschungen auf dem Gebiet der Zoologie und Anatomie sowie Botanik und Physiologie zu fördern. Insofern wirkte die Königlich-Preußische Akademie der Wissenschaften pragmatischen Tendenzen entgegen. Darüber hinaus bemühte sich Pringsheim, auch die Unterstützung einer gesamtdeutschen Autorität wie der Deutschen Zoologischen Gesellschaft zu gewinnen. Die Gesellschaft, die mit ihren eigenen Gründungsquerelen beschäftigt war und den erwähnten Streitigkeiten zwischen südwestdeutschen und Berliner „Oberzoologen“, einigte sich erst im Dezember 1890, also zu einem Zeitpunkt, als die Sache längst entschieden war, ihren Standpunkt dem Kultusministerium mitzuteilen. Inzwischen war die Angelegenheit der Stationsgründung zu einem Politikum herangereift. So äußerte der Heidelberger Ordinarius für Zoologie Otto Bütschli ${ }^{53}$ in einem Brief vom 6. 12. 1890 an Johann Spengel ${ }^{54}$, daß Eile geboten sei, da ja schon die Sozialdemokraten die Errichtung einer Station auf Helgoland empfehlen würden (vgl. A. Geus und H. Querner 1990 und pers. Mitteilung H. Querner). Bütschli war Vorsitzender der Sektion Zoologie der Versammlung der Naturforscher und Ärzte.

Als drittes Gremium nahm die Wissenschaftliche Kommission zur internationalen Meeresforschung Einfluß auf die Konzeption. Die Biologen waren sich darüber einig, daß die Aufgaben der künftigen Einrichtung über die Zoologie hinausgehen sollten. Nathanael Pringsheim übernahm es wiederum in einem Schreiben an Althoff, die Forderung der Fachleute nach einem breiten Forschungsgebiet zum Ausdruck zu bringen:

„Die Vorschläge gehen dahin, die Station nicht auf Zoologie zu beschränken, sondern ihr einen erweiterten Botanik und Physiologie einschließenden Umfang zu geben und wir schlagen deshalb den Namen biologische Station anstatt zoologische Station vor": (1).

Von Anfang an spielte in den Begründungen jedoch der Gedanke eine sehr wichtige Rolle, sich neben morphologischen, ontogenetischen und physiologischen Studien der Zoologie, auch praktischen Fragen zu widmen. Solche Fragen waren vor allem jene, die sich mit der Fischerei verbanden. Diese Gedanken wurden sowohl von staatlicher als auch von wirtschaftlicher Seite mit großem Interesse aufgenommen. Friedrich Heincke, der sich als Fischereizoologe für die Einrichtung der Station engagierte, stellte in Aussicht, daß sich das Vorhaben positiv auf die deutsche Fischereiwirtschaft auswirken werde. Geschickt führte er an, daß wirtschaftlicher Nutzen zu erwarten sei und daß Deutschland hinter anderen Ländern keinesfalls zurückbleiben dürfe. So heißt es in einem von ihm verfaßten Artikel:

„Allgemein wird auch in denjenigen Staaten, deren Fischerei viel höher entwickelt ist als die unsrige, anerkannt, daß zu einem zweckmäßigen und erfolgreichen Betrieb der Seefischerei genaue Kenntnis über die verschiedensten Verhältnisse der Meere, über Lebensweise, Nahrung, Wanderungen und Laichen der Fische, und über zahlreiche andere Dinge eine nothwendige Vorbedingung sind. Diese Verhältnisse, die noch nirgends auch nur in groben Umrissen bekannt sind, können nur durch regelmäßige wissenschaftliche Untersuchungen erforscht werden. Ueberall werden jetzt daher zu diesem Zweck Beobachtungsstationen an den Seeküsten gegründet, und die erhaltenen Resultate sind in manchen Fällen schon von erheblichem Einfluß auf die Fänge der von ihnen unterrichteten Fischer gewesen. Die Verwendung von Staatsmitteln zur Beförderung derartiger Untersuchungen wird sich in hohem Grade als produktiv erweisen und auf Grund solcher Arbeiten werden vielfach den Fischern an Ort und Stelle zweckmäßige Rathschläge über geeignete Fangplätze, Fanggeräthe usw. ertheilt werden können“ (vgl. Heincke 1888).

53 Otto Bütschli (1848-1920)

54 Johann Spengel (1852-1921) 
Allgemein gefaßt, so Heincke, dürfe eine solche Stationsgründung keine Rücksicht nehmen auf materielle Bedürfnisse und Ziele einzelner Menschen und Staaten. Institute, welche zu ihrer Förderung gegründet werden, wie z. B. die Zoologische Station zu Neapel, erfüllten ihren Zweck gerade deshalb so gut, weil sie international seien. In diesem Zusammenhang bezeichnete Heincke die Erforschung der Meere über die nationale Pflicht des vorwärtstreibenden Menschengeistes hinaus als gemeinsame Pflicht der Naturforscher aller Länder.

Pringsheims Argumentation ging in die gleiche Richtung. Auch er betonte, daß die in Aussicht stehenden Ergebnisse für alle sich anschließenden praktischen Fragen der Küstenfischerei und Nutzanwendung der Meerespflanzen und -tiere von nachhaltigem Gewinn seien. Er verknüpfte seine Behauptungen geschickt mit dem vaterländischen Gedanken, nicht nur der allgemeinen Wissenschaft überhaupt, sondern insbesondere der deutschen zu dienen. Darüber hinaus wurde aber auch die internationale Dimension deutlich gemacht und an ideelle Werte erinnert, denen die Wissenschaft sich verpflichtet fühlen müßte.

Nachdem mehrere positive Gutachten und Stellungnahmen vorlagen, konnte man dazu übergehen, Einzelheiten des Vorhabens wie Finanzierung, Größe, Personal usw. zu besprechen. Dies rief Anton Dohrn auf den Plan. Er wandte sich hilfesuchend an seinen Freund Robert von Keudell ${ }^{55}$. Von Keudell, bis 1887 deutscher Botschafter in Rom und enger Freund Dohrns, schrieb einen Brief an Althoff. Er bat ihn dringend, sich mit Anton Dohrn hinsichtlich der Größe der geplanten Station, ihrer Finanzierung und anderer Einzelheiten zu beraten. Althoff folgte dem Rat des Botschafters a. D., sich kritische Auffassungen anzuhören, und bat Anton Dohrn, der im preußischen Staat hohes Ansehen genoß und mit zahlreichen Orden und Titeln geehrt worden war, um ein Gutachten. Daraus entwickelte sich ein intensiver Briefwechsel. Bei der Betrachtung des Briefwechsels zwischen Dohrn und Althoff entsteht der Eindruck, daß Anton Dohrn sich nun, nachdem das Projekt nicht $\mathrm{zu}$ verhindern war, bemühte, seine Realisierung hinauszuzögern bzw. dafür zu sorgen, die Dimensionen in bescheidenem Rahmen zu halten. So führte er sehr diplomatisch aus, zu einer Meinungsäußerung nicht in der Lage zu sein, da er nicht einmal wisse, welche Funktion die künftige Einrichtung haben solle, ob sie ausschließlich Forschungszwecken dienen solle oder auch der Ausbildung. Er nutzte die Gelegenheit, seine prinzipiellen Bedenken gegen das Helgoländer Projekt zu wiederholen und Einwände geltend zu machen:

„Im allgemeinen kann ich nur Eurer Excellenz Aufmerksamkeit auf den Umstand richten, dass die Fauna von Helgoland nicht gerade mannigfaltig, die Möglichkeiten der Rohmaterialbeschaffung aber durch tägliches Fischen der häufigen Stürme halber sehr erschwert ist. Beides zusammen laesst es angezeigt erscheinen, in der Anlage der projektierten Station maessige Dimensionen nicht zu überschreiten“ (4).

Hierbei berief sich Dohrn auf negative Erfahrungen, die er angeblich während seines Aufenthaltes vor 25 Jahren auf Helgoland gesammelt hatte (vgl. I. Müller 1992). Indem er auf eine vermeintliche Armut der Fauna und Flora um Helgoland hinwies, hoffte Dohrn, die Realisierung des Projekts weiter zu verzögern. Er schlug vor, erst einmal zu prüfen, ob sich die Einrichtung einer Meeresstation überhaupt lohne, und riet, einen kenntnisreichen jungen Biologen damit zu beauftragen, ab Frühjahr 1891 Beschaffungsmöglichkeiten lebenden Materials auf Helgoland zu prüfen, quantitative Ermittlungen anzustellen und auch über die Witterung und den Zustand des Meeres täglich Buch zu führen. In $1{ }^{1 / 2}$ Jahren, so Dohrns Schätzung, würden genügend Daten zur Verfügung stehen.

55 Robert von Keudell (1824-1903) 
Alles deutet darauf hin, daß man auf Dohrns „Verzögerungstaktik“ nicht einging. Mehrere Monate später, im Mai 1891, machte Althoff den Vorschlag, ein Kuratorium zur Stationsgründung ins Leben zu rufen. Dieser Plan wurde von Wissenschaftlern wie Pringsheim und Clemens Hartlaub ${ }^{56}$ unterstützt. Dieses Kuratorium, bestehend aus - wie es hieß - vier in Berlin ansässigen Personen, - sollte die Angelegenheit betreiben. Der Zoologe Clemens Hartlaub war ein Schwiegersohn von Ehlers. Als Berater wollte man auch auswärtige Wissenschaftler hinzuziehen, vorgeschlagen wurden Hensen, Ehlers, Leuckart, Bütschli und Hertwig (3). Hensen, der anfänglich gegenüber Dohrn Bedenken geäußert hatte, befürwortete inzwischen die Gründung einer deutschen biologischen Station an der Nordsee, denn Althoff hatte Anton Dohrn dazu bewegen können, die von ihm und seinen Mitarbeitern entwickelten Präparationsmethoden für Meerestiere an deutsche Wissenschaftler weiterzugeben. Diese Methoden hielt Hensen für die Forschungsarbeit und den geplanten Versand von Versuchsmaterial an deutsche Universitäten für unentbehrlich (I. Müller, persönliche Mitteilung).

Zweimal, am 13., 27. Juni, und noch einmal am 13. Juli 1891 bat Althoff die engagierten Wissenschaftler ins Kultusministerium in Berlin zu weiteren Besprechungen. Noch war eine positive Entscheidung nicht gefallen, stand aber in Aussicht. Althoff verfolgte die Strategie, im Falle einer positiven Entscheidung gut vorbereitet zu sein. Deshalb ließ er sich Einzelheiten der Gründung der biologischen Station auf Helgoland erörtern (6).

Dennoch ließ Althoff die Gelehrten, die er mehrfach zu Gesprächen im Kultusministerium empfing, nie im Zweifel darüber, daß der Erfolg ihrer gemeinsamen Bemühungen unsicher war, da alles von der Bereitstellung der Finanzen abhängen würde. Diese Bemerkungen Althoffs ließen bei einigen Wissenschaftlern Zweifel darüber aufkommen, ob ein Vertreter des Kultusministeriums, zumal es sich nicht einmal um den Minister selbst, sondern „nur“ um einen Oberregierungsrat handelte, genügend Einfluß haben könnte, ihren Plan durchzusetzen. Es wurde darüber nachgedacht, höhergestellte Personen zu gewinnen. Wen, darüber gab es unterschiedliche Ansichten. So erwog Ernst Ehlers die Einreichung einer sogenannten "Immediatseingabe" an den Kaiser, Otto Bütschli hielt es hingegen für besser, sich gleich an den Reichskanzler zu wenden. Rudolf Leuckart wiederum wollte wissen, daß Bismarck mit der Angelegenheit verschont bleiben möchte. Trotzdem kam die Eingabe an Bismarck zustande, blieb aber unbeantwortet (vgl. Geus und Querner 1990). Ob Althoff davon erfuhr, ist nicht bekannt. Er forderte die Gelehrten zu einer Stellungnahme zum Vorschlag Dohrns auf, vor Einrichtung einer „provisorischen“ Anstalt auf Helgoland weitere Erhebungen anzustellen. Dies wurde abgelehnt.

Man war sich einig darüber, daß die Gründung eines provisorischen Instituts zum 1. April 1892 in Angriff genommen werden sollte. Das Institut sollte gleichzeitig wissenschaftliche wie praktische Ziele verfolgen. Finanzierungsfragen, z. B. das Problem, inwieweit Preußen und das Reich zu beteiligen seien, wurden zurückgestellt. Die laufenden Ausgaben für Miete der Gebäude, Sachkosten usw. und für Personalkosten wurden auf rund 50000 Mark veranschlagt. Was die Personalkosten betraf, so ging man zunächst davon aus, daß man außer dem Direktor zwei Abteilungsdirigenten oder Assistenten benötige, einen Verantwortlichen für die Beschaffung von Fischen etc., dazu einen Gehilfen, außerdem einen Maschinisten und einen Hausdiener. Was die einmaligen Ausgaben anging, darunter der Umbau des Hauses, die Anschaffung von Instrumenten, Booten und der Bibliothek, so wurden sie auf weitere 75000 Mark geschätzt. Heincke wurde im Ergebnis der Beratung beauftragt, in Zusammenarbeit mit den anderen anwesenden. Wissenschaftlern einen ausführlichen Plan aufzustellen. Außer ihm wurden Pringsheim, Schulze und Hartlaub in die

56 Clemens Hartlaub (1858-1928) 
Vorbereitungsarbeiten einbezogen. Heincke blieb mit Althoff in Verbindung und informierte ihn regelmäßig (122).

Im Auftrage Althoffs fuhren Nathanael Pringsheim und Franz Eilhard Schulze nach Helgoland, um vor Ort die Bedingungen für die Stationsgründung zu prüfen. Auch Clemens Hartlaub, der Bereitschaft gezeigt hatte, auf Helgoland als Kustos zu wirken, führte zusammen mit Heincke Ortsbesichtigungen durch. Jedoch noch im Juni 1891 war unsicher, wie Althoff in einem Brief an Clemens Hartlaub in Göttingen bemerkte, ob die Station tatsächlich gegründet werde, da die Mittelforderungen aus Helgoland sehr hoch wären (134).

Obwohl Althoff die Entscheidung der Regierung über die provisorische Stationsgründung bis zum September 1891 erwartet hatte, zögerte sich die Bestätigung hinaus, weil u. a. die Kostenfrage nicht geklärt war (129). So teilte Althoff Heincke noch am 7. September 1891 mit, daß zwar das Projekt gute Aussicht auf Bestätigung habe, aber von völliger Sicherheit noch nicht zu sprechen sei. Er riet Heincke, die Anmietung einer Wohnung auf der Insel noch etwas hinauszuschieben. Als Hauptproblem nannte Althoff die Beschaffung des Gebäudes. Ihm schwebte vor, ein geeignetes Haus zur unentgeltlichen, eventuell mietfreien Benutzung so lange zu bekommen, bis ein geeignetes Gebäude für die Station hergestellt sei.

Durch Vermittlung des Direktors des Berliner Aquariums, Otto Hermes ${ }^{57}$, der mit Heincke befreundet war, wurde dem Staat das Haus des Polizeisekretärs a. D. Botter angeboten. Es handelte sich um ein zweistöckiges Haus an der Jütlandterrasse. Als günstig erwies sich der Umstand, daß Botter nur Eigentümer auf etwa 60 Jahre war, der Grund und Boden gehörte, wie es hieß, der „Landschaft“ (7). Althoff ging davon aus, daß bei einer Auseinandersetzung zwischen Gemeinde und Staat der Staat gewinnen würde (128). Das Problem für das Kultusministerium war jedoch, daß Botter nicht vermieten, sondern verkaufen wollte. Das bedeutete, daß das Geld beschafft werden mußte. Botter verlangte 55000 Mark, ein Preis, der zwar hoch, aber durchaus angemessen erschien, denn es war auf Helgoland üblich, das Doppelte zu fordern. Althoff empfahl den Kauf, weil er davon überzeugt war, daß

„wer daher von Herrn Botter kauft, . . . rein geschäftlich betrachtet eine gute Kapitalanlage macht" (130).

Auch von anderer Seite wurden Angebote gemacht, so bot Gustav Rickmers sein Haus "Duke of Wellington" dem preußischen Fiskus zum Kauf an. Wie jedoch Althoff dem Gemeindevorsteher verbindlich mitteilte, sei davon keine Rede, daß der Staat auf das Rickmersche Haus reflektiere.

Als am 15. März 1892 vom Haus der Abgeordneten der bei der Budgetkommission eingereichte Antrag für den Erwerb des Gebäudes der Station und für Grund und Boden positiv entschieden werden konnte, stand dem Kauf des Hauses nichts mehr entgegen. Für die Entscheidung im Haus der Abgeordneten war der Umstand günstig, daß Otto Hermes zu den Abgeordneten gehörte. Er konnte in die Diskussion eingreifen und noch einmal den Sinn der Stationsgründung überzeugend erlüutern. Auch der Kultusminister, Graf von Zedlitz und Trützschler ${ }^{58}$, meldete sich mit einem Diskussionsbeitrag zu Wort. Er erklärte das staatliche Interesse an der Stationsgründung und stellte heraus, daß es nicht nur um ein wissenschaftliches und wirtschaftliches Anliegen gehe, sondern da $\beta$ man damit auch die Helgoländer Bevölkerung durch Schaffung von Arbeitsplätzen unterstütze. Er berief sich hierbei auf die Abschiedsrede des scheidenden englischen Gouverneurs, der die Errichtung einer Zoologischen Station den Helgoländern empfohlen habe.

57 Otto Hermes (1838-1910)

58 Graf Robert von Zedlitz und Trützschler (1837-1914) 


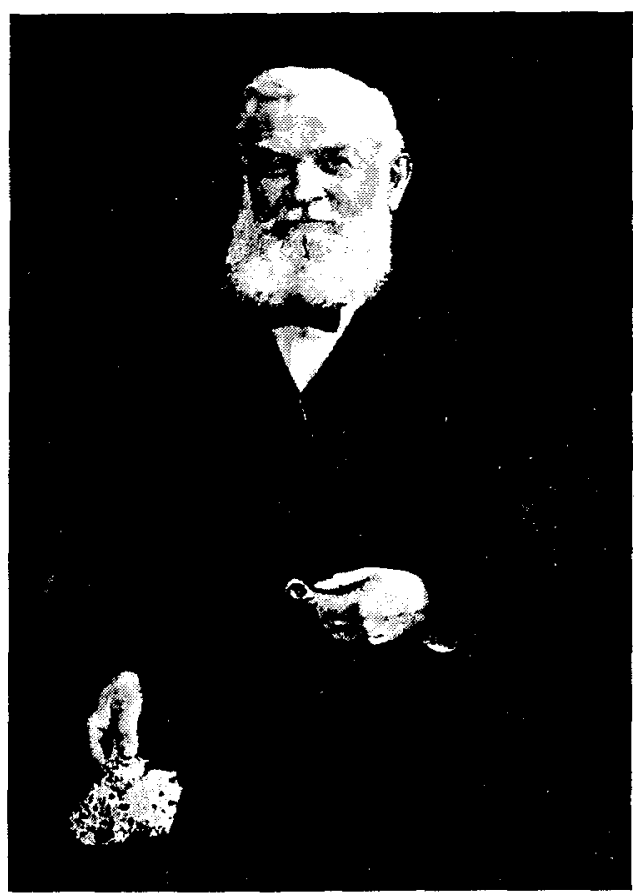

Abb. 7. Franz Eilhard Schulze (1840 -1921). Archiv der Akademie der Wissenschaften, Berlin, Fotosammlung.

Die Verhandlungen mit dem Verkäufer des Gebäudes, dem Polizeisekretär a. D. Paul J. Botter, führte Friedrich Heincke persönlich. Er war bereits seit zwei Jahren mit Botter im Gespräch, auch Meßbildaufnahmen der künftigen Baustelle waren auf seine Veranlassung hin bereits 1890 angefertigt worden. In einer Notiz an Althoff gab Heincke eine ausführliche Schilderung der örtlichen Verhältnisse. Bemerkenswert ist, daß aus Heinckes Beschreibungen schon die Absicht deutlich wurde, in naher Zukunft weitere Gebäude, darunter die Post, für die Anstalt zu erwerben (131).

Am 29. April 1892 waren die Verhandlungen über den Erwerb des Botterschen Grundstücks so weit gediehen, daß ein Vertragsentwurf aufgesetzt werden konnte. Den neuen Besitzern wurde erlaubt, mit den Bauarbeiten zu beginnen. Zur Vertragsunterzeichnung kam es offiziell erst am 31. Mai 1892. Der Kaufpreis betrug 55000 Mark.

Mit dem Vertrag erwarb die Königliche Unterrichtsverwaltung, vertreten durch Heincke, das auf Kronland erbaute, zwischen Kaiserlicher Post und Villa Franz befindliche Gebäude und das Grundstück. Hier stand somit das erste Gebäude der Königlichen Biologischen Anstalt auf Helgoland.

Der Vertrag wurde am 11. Juni 1892 vom Ministerium bestätigt. Eine Gründungsurkunde existiert nicht. Es ist auch nicht bekannt, ob eine Einweihungsfeier stattfand. Friedrich Heincke, der zunächst als kommissarischer Direktor eingesetzt wurde, erhielt seine Berufungsurkunde als Direktor am 15. 6. 1892. Dieses Datum betrachtet die Nathanael-Pringsheim-Gesellschaft als Gründungsdatum der Königlichen Biologischen Anstalt zu Helgoland. 